\title{
Wissenschaftspreis 2017 - Ausschreibung
}

Die Deutsche Gesellschaft für Ultraschall in der Medizin (DEGUM) vergibt einen Preis für die hervorragende wissenschaftliche Bearbeitung eines Themas auf dem Gebiet des medizinischen Ultraschalls. Der Preis ist mit 5.000 ,-€ dotiert und kann geteilt werden. Es können sich jüngere Wissenschaftler (Geburtsjahr 1977 oder jünger) bewerben, die eine oder mehrere, richtungsweisende Arbeiten zu einem Thema publiziert haben. Es kann auch eine Habilitationsschrift eingereicht werden, sofern diese auf hochrangig publizierten Originalarbeiten beruht.

Der Wissenschaftsbeirat der DEGUM bewertet die Arbeit auf der Grundlage von zwei Gutachten und entscheidet über die Preisvergabe, seine Entscheidung ist endgültig und nicht anfechtbar.

Die Bewerbung ist auf elektronischem Weg bis zum 30.04.2017 an die DEGUM-Geschäftsstelle (geschaeftsstelle@degum.de) zu richten. Der Bewerbung sollen die relevanten Publikationen, ein Lebenslauf des
Bewerbers sowie eine kurze Zusammenfassung der Arbeiten (nicht mehr als drei DIN A4-Seiten), jeweils in digitaler Form und ebenfalls auf elektronischem Weg, beigefügt werden. Des Weiteren ist vom Antragsteller eine formlose Erklärung beizufügen, dass die vorgelegte( $n$ ) Arbeit(en) für keinen andersartigen Preis eingereicht wurde(n).

Die Preisverleihung erfolgt während des Dreiländertreffens 2017 in Linz / Österreich (11.-14.10.2017). Die persönliche Anwesenheit der Preisträgerin/des Preisträgers ist obligat. Von ihr/ihm wird erwartet, dass sie/er die mit dem DEGUM-Preis ausgezeichnete Arbeit während des Dreiländertreffens in Form eines Vortrags vorstellt.

Berlin im November 2016

PD Dr. med. Kai-Sven Heling, Präsident der DEGUM

Prof. Dr. med. Andreas Hagendorff, Sekretär der DEGUM 